\title{
IMA Commission on New Minerals, Nomenclature and Classification (CNMNC)
}

\author{
NEWSLETTER 40
}

\section{New minerals and nomenclature modifications approved in 2017}

\section{U. Hålenius ${ }^{1}$ (Chairman CNMnC), F. Hatert ${ }^{2}$ (Vice-Chairman CNMNC), M. Pasero ${ }^{3}$ (Vice-Chairman} CNMNC) and S. J. Mills ${ }^{4}$ (Secretary, CNMNC)

1 Department of Geosciences, Naturhistoriska Riksmuseet, Box 50007, SE-104 05 Stockholm, Sweden ulf.halenius@nrm.se

2 Laboratoire de Minéralogie, Université de Liège, B-4000 Liège, Belgium - fhatert@ulg.ac.be

3 Dipartimento di Scienze della Terra, Università di Pisa, Via Santa Maria 53, I-56126 Pisa, Italy marco.pasero@unipi.it

4 Geosciences, Museum Victoria, PO Box 666, Melbourne, Victoria 3001, Australia - smills@museum.vic.gov.au

The information given here is provided by the IMA Commission on New Minerals, Nomenclature and Classification for comparative purposes and as a service to mineralogists working on new species.

Each mineral is described in the following format:

Mineral name, if the authors agree on its release prior to the full description appearing in press Chemical formula

Type locality

Full authorship of proposal

E-mail address of corresponding author

Relationship to other minerals

Crystal system, Space group; Structure determined, yes or no

Unit-cell parameters

Strongest lines in the powder X-ray diffraction pattern

Type specimen repository and specimen number

Citation details for the mineral prior to publication of full description

Citation details concern the fact that this information will be published in the Mineralogical Magazine on a routine basis, as well as being added month by month to the Commission's web site.

It is still a requirement for the authors to publish a full description of the new mineral.

NO OTHER INFORMATION WILL BE RELEASED BY THE COMMISSION 


\section{NEW MINERAL PROPOSALS APPROVED IN OCTOBER 2017}

\section{IMA No. 2017-064}

Silesiaite

$\mathrm{Ca}_{2} \mathrm{Fe}^{3+} \mathrm{Sn}\left(\mathrm{Si}_{2} \mathrm{O}_{7}\right)\left(\mathrm{Si}_{2} \mathrm{O}_{6} \mathrm{OH}\right)$

In a pegmatite of the Karkonosze granite, Szklarska Poręba Huta quarry, Lower Silesia, Poland (50.82778 N, 15.48944 E)

Adam Pieczka*, Chi Ma, George R. Rossman, R. James Evans, Lee A. Groat and Bożena Gołębiowska

*E-mail: pieczka@agh.edu.pl

The $\mathrm{Fe}^{3+}$ analogue of kristiansenite

Triclinic: $C 1$

$a=10.028(1), \quad b=8.408(1), \quad c=13.339(2) \AA$, $\alpha=90.01(1), \beta=109.10(1), \gamma=90.00(1)^{\circ}$

$9.147(100), \quad 8.408(12), \quad 6.607(64), \quad 5.195(6)$, 4.413(10), 3.312(7), 3.151(12), 3.095(9)

Type material is deposited in the collections of the Mineralogical Museum, University of Wrocław, Cybulskiego 30, 50-205 Wrocław, Poland, catalogue number MMWr IV7929

How to cite: Pieczka, A., Ma, C., Rossman, G.R., Evans, R.J., Groat, L.A. and Gołebiowska, B. (2017) Silesiaite, IMA 2017-064. CNMNC Newsletter No. 40, December 2017, page 1578; Mineralogical Magazine, 81, 1577-1581.

\section{IMA No. 2017-065}

Meitnerite

$\left(\mathrm{NH}_{4}\right)\left(\mathrm{UO}_{2}\right)\left(\mathrm{SO}_{4}\right)(\mathrm{OH}) \cdot 2 \mathrm{H}_{2} \mathrm{O}$

Green Lizard Mine, White Canyon mining district, San Juan Co., Utah, USA (37 $34^{\prime} 37.10^{\prime \prime}$ $\mathrm{N}, 110^{\circ} 17^{\prime} 52.80^{\prime \prime} \mathrm{W}$ )

Anthony R. Kampf*, Jakub Plášil, Barbara P. Nash and Joe Marty

*E-mail: akampf@nhm.org

Structurally related to johannite

Triclinic: $P \overline{1}$; structure determined

$a=6.7964(2), \quad b=8.0738(3), \quad c=9.2997(7) \AA$,

$\alpha=113.284(8), \beta=99.065(7), \gamma=105.289(7)^{\circ}$

7.15(100), 6.36(30), 5.85(36), 5.038(21), 3.569

(19), 3.451(18), 3.340(20), 3.075(21)

Type material is deposited in the mineralogical collections of the Natural History Museum of Los Angeles County, 900 Exposition Boulevard, Los Angeles, CA 90007, USA, catalogue number 66623

How to cite: Kampf, A.R., Plášil, J., Nash, B.P. and Marty, J. (2017) Meitnerite, IMA 2017-065.
CNMNC Newsletter No. 40, December 2017, page 1578; Mineralogical Magazine, 81, $1577-1581$.

IMA No. 2017-066

Marchettiite

$\mathrm{C}_{5} \mathrm{H}_{7} \mathrm{~N}_{5} \mathrm{O}_{3}$

SW slope of Mount Cervandone, Devero valley, Baceno, Verbano-Cusio-Ossola, Piedmont, Italy Alessandro Guastoni, Fabrizio Nestola, Paolo Gentile, Federico Zorzi, Sergio Andò, Alessandra Lorenzetti and Vittorio Mattioli

*E-mail: alessandro.guastoni@unipd.it

Known synthetic analogue

Triclinic: $P \overline{1}$ or $P 1$

$a=3.6533(2), b=10.2046(7), c=10.5837(7) \AA$, $\alpha=113.809(5), \beta=91.313(8), \gamma=92.44(1)^{\circ}$

$9.784(50), 8.663(80), 5.659(100), 4.614(50)$, 3.443(100), 3.241(70), 3.158(100), 3.004(60)

Type material is deposited in the collections of the Museum of Mineralogy, University of Padova, Via Giotto 1, I-35122, Padova, Italy, catalogue number MMP M17892

How to cite: Guastoni, A., Nestola, F., Gentile, P., Zorzi, F., Andò, S., Lorenzetti, A. and Mattioli, V. (2017) Marchettiite, IMA 2017066. CNMNC Newsletter No. 40, December 2017, page 1578; Mineralogical Magazine, 81, $1577-1581$.

\section{IMA No. 2017-068}

Vestaite

$\left(\mathrm{Ti}^{4+} \mathrm{Fe}^{2+}\right) \mathrm{Ti}_{3}^{4+} \mathrm{O}_{9}$

NWA 8003 meteorite, fall in an unknown locality in Morocco

Run-Lian Pang, Dennis Harries, Kilian Pollok, Ai-Cheng Zhang* and Falko Langenhorst*

*E-mail: aczhang@nju.edu.cn, falko.langenhorst@ uni-jena.de

The $\mathrm{Ti}^{4+}-\mathrm{Fe}^{2+}$ analogue of schreyerite

Monoclinic: $C 2 / c$

$a=17.03(2), \quad b=4.98(1), \quad c=7.08(1) \quad \AA$,

$\beta=106.3(2)^{\circ}$

4.084(44), 3.398(60), 2.880(77), 2.732(100), 2.425(42), 1.705(38), 1.694(50), 1.643(48),

Type material is deposited in the mineralogical collections of the Friedrich Schiller University Jena, Sellierstrasse 6, 07745 Jena, Germany, catalogue numbers 42073 and 42074

How to cite: Pang, R.-L., Harries, D., Pollok, K., Zhang, A.-C. and Langenhorst, F. (2017) Vestaite, 
IMA 2017-068. CNMNC Newsletter No. 40, December 2017, page 1578; Mineralogical Magazine, 81, 1577-1581.

IMA No. 2017-070

Hjalmarite

$\mathrm{Na}(\mathrm{NaMn}) \mathrm{Mg}_{5} \mathrm{Si}_{8} \mathrm{O}_{22}(\mathrm{OH})_{2}$

Långban Fe-Mn-(Ba-As-Pb-Sb) deposit,

Filipstad district, Värmland, Sweden $\left(59.86^{\circ} \mathrm{N}\right.$, 14.26 ${ }^{\circ} \mathrm{E}, 215 \mathrm{~m}$ a.s.1.)

Dan Holtstam*, Fernando Cámara, Henrik

Skogby and Andreas Karlsson

*E-mail: dan.holtstam@vr.se

Amphibole supergroup

Monoclinic: $C 2 / \mathrm{m}$; structure determined

$a=9.9113(3), \quad b=18.1361(4), \quad c=5.2831(5)$,

$\beta=103.658(5)^{\circ}$

$8.50(44), \quad 3.302(40), \quad 3.164(100), \quad 2.837(50)$,

2.727(30), 2.183(17), 1.670(34), 1.447(32)

Type material is deposited in the mineralogical collections of the Department of Geosciences, Swedish Museum of Natural History, Box 50007, SE-10405 Stockholm, Sweden, collection number NRM \#g14150

How to cite: Holtstam, D., Cámara, F., Skogby, H. and Karlsson, A. (2017) Hjalmarite, IMA 2017-070. CNMNC Newsletter No. 40, December 2017, page 1579; Mineralogical Magazine, 81, 1577-1581.

\section{IMA No. 2017-071}

Dekatriasartorite

$\mathrm{TlPb}_{58} \mathrm{As}_{97} \mathrm{~S}_{204}$

Lengenbach quarry, Binntal, Wallis, Switzerland $\left(46^{\circ} 21^{\prime} 54^{\prime \prime} \mathrm{N}, 8^{\circ} 13^{\prime} 15^{\prime \prime} \mathrm{E}\right)$

Dan Topa*, Berthold Stoeger, Emil Makovicky and Chris Stanley

*E-mail: dan.topa@nhm-wien.ac.at

Sartorite homologous series

Monoclinic: $P 2_{1} / c$; structure determined

$a=54.576(5), b=7.8947(6), c=20.102(16) \AA$, $\beta=78.153(1)^{\circ}$

9.84(58), 3.870(69), 3.522(100), 3,464(52),

2.966(64), 2.955(87), 2.762(71), 2.758(70)

Type material is deposited in the reference collections of the Naturhistorisches Museum Wien, Burgring 7, A-1010 Wien, Austria, catalogue number $\mathrm{N} 9863$

How to cite: Topa, D., Stoeger, B., Makovicky, E. and Stanley, C. (2017) Dekatriasartorite, IMA 2017-071. CNMNC Newsletter No. 40,
December 2017, page 1579; Mineralogical Magazine, 81, 1577-1581.

\section{IMA No. 2017-072}

Oberthürite

$\mathrm{Rh}_{3} \mathrm{Ni}_{32} \mathrm{~S}_{32}$

Marathon deposit, Coldwell Complex, Ontario, Canada $\left(48^{\circ} 48^{\prime} 7^{\prime \prime} \mathrm{N}, 86^{\circ} 18^{\prime} 35^{\prime \prime} \mathrm{W}\right)$

Andrew M. McDonald*, Ingrid M. Kjarsgaard, Kirk C. Ross, Doreen E. Ames, Louis J. Cabri and David J. Good

*E-mail: amcdonald@laurentian.ca

Pentlandite group

Cubic: $F \overline{4} 3 m$; structure determined $a=10.066(5) \AA$

$3.060(100), \quad 2.929(18), 1.952(39), 1.792(74)$, 1.543(9), 1.318(15), 1.031(30), 0.976(10)

Type material is deposited in the mineralogical collections of the Canadian Museum of Nature, Gatineau, Quebec, Canada, catalogue number 87251

How to cite: McDonald, A.M., Kjarsgaard, I.M., Ross, K.C., Ames, D.E., Cabri, L.J. and Good, D. J. (2017) Oberthürite, IMA 2017-072. CNMNC Newsletter No. 40, December 2017, page 1579; Mineralogical Magazine, 81, 1577-1581.

\section{IMA No. 2017-073}

Ammoniozippeite

$\left(\mathrm{NH}_{4}\right)_{2}\left[\left(\mathrm{UO}_{2}\right)_{2}\left(\mathrm{SO}_{4}\right) \mathrm{O}_{2}\right] \cdot \mathrm{H}_{2} \mathrm{O}$

Blue Lizard Mine, Red Canyon, White Canyon mining district, San Juan Co., Utah, USA (37 $33^{\prime}$ $\left.26^{\prime \prime} \mathrm{N}, 110^{\circ} 17^{\prime} 44^{\prime \prime} \mathrm{W}\right)$; Burro mine, Slick Rock district, San Miguel Co., Colorado, USA (38 $2^{\prime}$ $42^{\prime \prime} \mathrm{N} 108^{\circ} 53^{\prime} 23^{\prime \prime} \mathrm{W}$ )

Anthony R. Kampf*, Jakub Plášil, Travis A. Olds, Barbara P. Nash and Joe Marty *E-mail: akampf@nhm.org The $\left(\mathrm{NH}_{4}\right)$ analogue of zippeite Orthorhombic: $C \mathrm{cmb}$; structure determined $a=8.7944(3), b=14.3296(7), c=17.172(1) \AA$ $7.17(100), \quad 4.270(13), \quad 3.670(14), \quad 3.580(21)$, 3.489(42), 3.138(63), 1.750(14), 1.697(18)

Type material is deposited in the mineralogical collections of the Natural History Museum of Los Angeles County, 900 Exposition Boulevard, Los Angeles, CA 90007, USA, catalogue number 66625 (Burro - holotype) and 66626 (Blue Lizard - cotype)

How to cite: Kampf, A.R., Plášil, J., Olds, T.A., Nash, B.P. and Marty, J. (2017) 
Ammoniozippeite, IMA 2017-073. CNMNC Newsletter No. 40, December 2017, page 1579; Mineralogical Magazine, 81, 1577-1581.

\section{IMA No. 2017-074}

Hydroxynatropyrochlore $(\mathrm{Na}, \mathrm{Ca}, \mathrm{Ce})_{2} \mathrm{Nb}_{2} \mathrm{O}_{6}(\mathrm{OH})$

Phoscorite-carbonatite pipe, Kovdor massif, Murmansk Region, Russia

Gregory Y. Ivanyuk, Victor N. Yakovenchuk, Taras L. Panikorovskii, Nataliya Konoplyova, Yakov A. Pakhomovsky, Ayya V. Bazai, Vladimir N. Bocharov and Sergey V. Krivovichev

*E-mail: s.krivovichev@spbu.ru

Pyrochlore supergroup

Cubic: $F d \overline{3} m$; structure determined

$\mathrm{a}=10.3276(5) \AA$

$5.96(47), \quad 3.110(30), \quad 2.580(100), \quad 2.368(19)$, 1.987(6), 1.826(25), 1.746(3), 1.556(14)

Type material is deposited in the collections of the Mineralogical Museum, Saint-Petersburg State University, University Emb. 7/9, St. Petersburg 199034, Russia, catalogue number 1/19679

How to cite: Ivanyuk, G.Y., Yakovenchuk, V.N., Panikorovskii, T.L., Konoplyova, N., Pakhomovsky, Y.A., Bazai, A.V., Bocharov, V.N. and Krivovichev, S.V. (2017) Hydroxynatropyrochlore, IMA 2017-074. CNMNC Newsletter No. 40, December 2017, page 1580; Mineralogical Magazine, 81, 1577-1581.

\section{IMA No. 2017-075}

Hydroxylpyromorphite

$\mathrm{Pb}_{5}\left(\mathrm{PO}_{4}\right)_{3}(\mathrm{OH})$

Copps mine, Gogebic Co., Michigan, USA ( $46^{\circ}$ $27^{\prime} 24^{\prime \prime} \mathrm{N}, 89^{\circ} 39^{\prime} 38^{\prime \prime} \mathrm{W}$ )

Travis A. Olds*, Shawn M. Carlson, Anthony R. Kampf, John Rakovan, Cullen Laughlin-Yurs, Peter C. Burns and Owen P. Mills

*E-mail: tolds@nd.edu

Apatite supergroup

Hexagonal: $P 6_{3} / m$; structure determined $a=9.787(1), c=7.307(1) \AA$

$4.079(18), \quad 3.359(29), \quad 3.207(21), \quad 2.934(100)$, 2.035(21), 1.942(27), 1.834(25), 1.592(17)

Type material is deposited in the mineralogical collections of the Natural History Museum of Los Angeles County, 900 Exposition Boulevard, Los Angeles, CA 90007, USA, catalogue number 66627 How to cite: Olds, T.A., Carlson, S.M., Kampf, A.R., Rakovan, J., Laughlin-Yurs, C., Burns, P.C. and Mills, O.P. (2017) Hydroxylpyromorphite, IMA 2017-075. CNMNC Newsletter No. 40, December 2017, page 1580; Mineralogical Magazine, 81, 1577-1581.

\section{NEW MINERAL PROPOSALS APPROVED IN NOVEMBER 2017}

\section{IMA No. 2017-076}

Manganflurlite

$\mathrm{ZnMn}_{3}^{2+} \mathrm{Fe}^{3+}\left(\mathrm{PO}_{4}\right)_{3}(\mathrm{OH})_{2}\left(\mathrm{H}_{2} \mathrm{O}\right)_{7} \cdot 2 \mathrm{H}_{2} \mathrm{O}$

Hagendorf-Süd pegmatite, Hagendorf, Oberpfalz, Bavaria, Germany $\left(49^{\circ} 39^{\prime} 1^{\prime \prime} \mathrm{N}, 12^{\circ} 27^{\prime} 35^{\prime \prime} \mathrm{E}\right)$

Anthony R. Kampf*, Ian E. Grey, Colin M. MacRae and Erich Keck

*E-mail: akampf@nhm.org

The Mn analogue of flurlite

Monoclinic: $P 2_{1} / \mathrm{m}$; structure determined $a=6.4546(8), b=11.1502(9), c=13.1630(10) \AA$, $\beta=99.829(5)^{\circ}$

12.89(100), 8.43(38), 6.14(22), 5.57(28), 4.241 (26), 3.206(29), 2.776(95), 2.713(27)

Type material is deposited in the mineralogical collections of the Natural History Museum of Los Angeles County, 900 Exposition Boulevard, Los Angeles, CA 90007, USA, catalogue number 66682

How to cite: Kampf, A.R., Grey, I.E., MacRae, C.M. and Keck, E. (2017) Manganflurlite, IMA 2017-076. CNMNC Newsletter No. 40, December 2017, page 1580; Mineralogical Magazine, 81, 1577-1581.

IMA No. 2017-077

Ammoniomathesiusite

$\left(\mathrm{NH}_{4}\right)_{5}\left(\mathrm{UO}_{2}\right)_{4}\left(\mathrm{SO}_{4}\right)_{4}\left(\mathrm{VO}_{5}\right) \cdot 4 \mathrm{H}_{2} \mathrm{O}$

Burro mine, Slick Rock district, San Miguel Co., Colorado, USA $\left(38^{\circ} 2^{\prime} 42^{\prime \prime} \mathrm{N} 108^{\circ} 53^{\prime} 23^{\prime \prime} \mathrm{W}\right)$

Anthony R. Kampf*, Jakub Plášil, Barbara P. Nash and Joe Marty

*E-mail: akampf@nhm.org

The $\left(\mathrm{NH}_{4}\right)$ analogue of mathesiusite

Tetragonal: $P 4 / n$; structure determined $a=14.9405(9), c=7.1020(5) \AA$

10.57(46), 7.10(62), 6.41(100), 4.71(27), 3.575 (25), 3.460(26), 3.340(35), 3.226(44)

Type material is deposited in the mineralogical collections of the Natural History Museum of Los Angeles County, 900 Exposition Boulevard, Los 
Angeles, CA 90007, USA, catalogue number 67248 (holotype), 67249, 67250 and 67251 (cotype)

How to cite: Kampf, A.R., Plášil, J., Nash, B.P. and Marty, J. (2017) Ammoniomathesiusite, IMA 2017-077. CNMNC Newsletter No. 40, December 2017, page 1580; Mineralogical Magazine, 81, 1577-1581.

IMA No. 2017-079

Janchevite

$\mathrm{Pb}_{7} \mathrm{~V}^{5+}\left(\mathrm{O}_{8.5} \square_{0.5}\right) \mathrm{Cl}_{2}$

Kombat mine, Grootfontein district, Otjozondjupa region, Namibia $\left(19^{\circ} 46^{\prime} 59^{\prime \prime} \mathrm{S}, 18^{\circ}\right.$ $\left.1^{\prime} 0^{\prime \prime} \mathrm{E}\right)$

Nikita V. Chukanov*, Diana O. Nekrasova, Oleg I. Siidra, Yury S. Polekhovsky and Igor V. Pekov

*E-mail: nikchukanov@yandex.ru

The $\mathrm{V}$ analogue of asisite

Tetragonal: $14 / \mathrm{mmm}$

$a=3.9591(5), c=22.6897(3) \AA$

$3.889(24), \quad 3.501(31), \quad 2.979(86), \quad 2.833(25)$,

2.794(100), 1.992(26), 1.988(49), 1.649(46)

Type material is deposited in the collections of the

Fersman Mineralogical Museum, Russian Academy of Sciences, Moscow, Russia, registration number 5105/1

How to cite: Chukanov, N.V., Nekrasova, D.O., Siidra, O.I., Polekhovsky, Y.S. and Pekov, I.V. (2017) Janchevite, IMA 2017-079. CNMNC Newsletter No. 40, December 2017, page 1581; Mineralogical Magazine, 81, 1577-1581.

IMA No. 2017-080

Guite

$\mathrm{Co}^{2+} \mathrm{Co}_{2}^{3+} \mathrm{O}_{4}$

Sicomines copper-cobalt mine, ca. $11 \mathrm{~km} \mathrm{SW}$ of Kolwezi City, Democratic Republic of Congo $\left(10^{\circ} 44^{\prime} 17.4^{\prime \prime} \mathrm{S}, 25^{\circ} 22^{\prime} 50.4^{\prime \prime} \mathrm{E}\right)$

Zhilan Lei, Xinghai Chen, Jianxiong Wang*, Jigui Zhang, Yingchun Huang, Zhiyu Lu and Fangfang Du

*E-mail: wangerlang@163.com

Spinel supergroup

Cubic: $F d 3 m$

$a=8.0848(1) \AA$

$2.862(18), \quad 2.440(100), 2.335(10), 2.023(25)$,

1.556(26), 1.430(38), 1.052(10), 0.825(9)

Type material is deposited in the mineralogical collections of the Geological Museum of China, 16 Yangrou Hutong, Xisi, Beijing 100031, People's Republic of China, catalogue number M13711
How to cite: Lei, Z., Chen, X., Wang, J., Zhang, J., Huang, Y., Lu, Z. and Du, F. (2017) Guite, IMA 2017-080. CNMNC Newsletter No. 40, December 2017, page 1581; Mineralogical Magazine, 81, 1577-1581.

\section{IMA No. 2017-081}

Wuyanzhiite

$\mathrm{Cu}_{2} \mathrm{~S}$

Bofang copper mine (-65 m level), ca. $40 \mathrm{~km} \mathrm{~S}$ of Hengyang City, Hunan Province, China $\left(26^{\circ}\right.$ $32^{\prime} 24.4^{\prime \prime} \mathrm{N}, 112^{\circ} 26^{\prime} 53.5^{\prime \prime} \mathrm{E}$ )

Xiangping $\mathrm{Gu}^{*}$, Xiangyang Shi, Hexiong Yang, Anhuai Lu, Yongjun Shao, Qian Chen and Zhongfa Liu

*E-mail: guxp2004@163.com

A dimorph of chalcocite

Tetragonal: $P 4_{3} 2_{1} 2$

$a=4.0008(1), c=11.2671(9) \AA$

2.833(24), 2.746(100), 2.304(97), 2.262(28), 1.998(62), 1.967(29), 1.887(26), 1.704(28)

Type material is deposited in the mineralogical collections of the Geological Museum of China, 16 Yangrou Hutong, Xisi, Beijing 100031, People's Republic of China, catalogue number M13712

How to cite: Gu, X., Shi, X., Yang, H., Lu, A., Shao, Y., Chen, Q. and Liu, Z. (2017) Wuyanzhiite, IMA 2017-081. CNMNC Newsletter No. 40, December 2017, page 1581; Mineralogical Magazine, 81, 1577-1581.

\section{NOMENCLATURE PROPOSALS APPROVED IN OCTOBER 2017}

\section{Gatelite supergroup}

A new classification and nomenclature scheme has been approved for the minerals of the gatelite supergroup. The supergroup is divided into three groups: gatelite group, västmanlandite group, alnaperbøeite group.

\section{Schoonerite group}

A new classification and nomenclature scheme has been approved for the minerals of the schoonerite group. Currently the group includes three mineral species: schoonerite, wilhelmgümbelite, and schmidite. 